\title{
The Operating System for the Computer of the 21st Century
}

\author{
Christian Becker \\ Chair for Information Systems II, Mannheim University, Germany \\ christian.becker@uni-mannheim.de
}

\begin{abstract}
Weiser's vision of Ubiquitous Computing has inspired many researchers and an active research community has been established in the past years. Various aspects have been investigated from novel user interfaces, applications to specialized hardware devices and system software helping application developers utilizing the capabilities of a smart world. In my talk I want to address the challenges researchers have addressed in the past. Based on a number of products and prototypes I will identify the problems where concepts and solutions exist and will also highlight open research challenges with a focus on adaptation and self-organization.
\end{abstract}

Keywords: Ubiquitous computing; operating system, adaptation. 